\title{
Study of filament power based on TMS320F28035
}

\author{
FAN Sheng Wen ${ }^{1,}$ a LV Guan Da ${ }^{1, b}$ and LI Lei ${ }^{1, c}$ \\ ${ }^{1}$ Power Electronics \& Motor Drivers Engineering Research Center, North China \\ University of Technology, Beijing 100144,China \\ afanshengwen@ncut.edu.cn, ${ }^{\mathrm{b}}$ Ivguanda@sohu.com, ${ }^{\mathrm{C}} 59008425 @$ sohu.com
}

Keywords: Electron Beam Melting Furnace; filament power; MATLAB Simulation

\begin{abstract}
The industrial frequency voltage regulator is used for the traditional filament power of electron beam melting furnace, it cannot realize the filament current closed-loop adjustment. To solve these problems, a 20A/300W filament power of electron beam melting furnace based on TMS320F28035 is designed in the paper. The MATLAB visualization simulation tool is used to establish the simulation model. The experiment results based on the simulation results are verified that designed parameters are correct, at the same time realizing the closed-loop control of the filament.
\end{abstract}

\section{Introduction}

With the rapid development of scientific and technology, more and more high purity and alloy are more needed by modern industrial. Conventional method cannot satisfy the production requirements. The vacuum electron beam melting is an effective purification method, especially effective for the metal and alloy which has lower vapor pressure under melting temperature.

Electron beam melting furnace is mainly used for smelting and refining high temperature refractory metals. Electron beam melting is working under high vacuum. The electronic gun focuses dozens to hundreds of kilowatts of high-energy electron beam on the $1 \mathrm{~cm}^{2}$ focus produce high temperature to melt metals, volatile impurities and achieve the purpose of purification. The entire electron beam melting furnace power supply includes three parts, high voltage power supply, bombardment power supply and filament power supply. The three power supplies have different functions. Filament power supply is usually used for heating filament by dozens of AC currents to make the filament produces high temperature and achieve the purpose of producing a large number of electrons around. Bombardment power supply is a $5 \mathrm{kV}$ DC negative high voltage that added on the filament and the cathode board which can make the electrons that filament produces bombarded to the cathode board by DC negative high voltage. Acceleration power supply is as high as 20 to $30 \mathrm{kV}$ added between the anode and cathode board which can produce high power electron beam and launch them to the metal need to be smelted to smelting and refining refractory metal.

This article makes a filament power supply prototype by using STM320F28035 as the controller for digital control, realizes the high frequency design of filament power supply for the melting furnace and realizes the filament current closed loop function.

\section{The main circuit principle and parameter design}

The main function of filament power supply in an electron beam melting furnace power supply is heating filament to make the filament produces electrons provided to high voltage power supply to accelerate the electrons. Then the high voltage electron beam produced by accelerated electrons can smelt metal.

2.1 The main circuit topology structure

The topology of the electron beam melting furnace filament power supply is shown in figure 1. Main circuit is composed of the following parts. Q1, D5, L1, C2 are BUCK converter part, Q2 Q5 is full bridge inverter unit made of four MOSFET. T1 is high frequency step-down transformer. S1 is 
current hall sensor. R1 is filament load. The transformer needs to be insulated when filament power supply and bombardment power supply are in series connection.

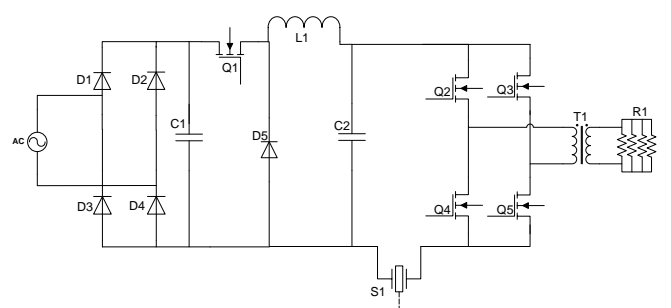

Fig.1 Topology of electron beam melting furnace filament power supply

BUCK converter is a kind of single pipe unfenced dc converter that output voltage is equal to or less than the input voltage as shown in figure 2. BUCK converter is composed of main circuit switch tube $\mathrm{T}$, diode $\mathrm{D}$, output filter inductance $\mathrm{L}$ and output filter capacitor $\mathrm{C}$. The power supply of this circuit is voltage source and the load is current source. The circuit can complete the function of convert the dc input voltage Ui into lower dc voltage Uo. BUCK converter has current continuous (CCM) and discontinuous inductor current (DCM) two modes. Load current can be continuous and ripple can be small by concatenating large inductance. At the same time, the core requirement of filament power supply is having stable output current, so the article takes the CCM mode.

The final stage of BUCK convertor adopts full bridge mode. DC voltage will be inverted into high frequency ac square wave transmitted to isolation transformer. The filament current can be adjusted by controlling the change of the transformer ratio.

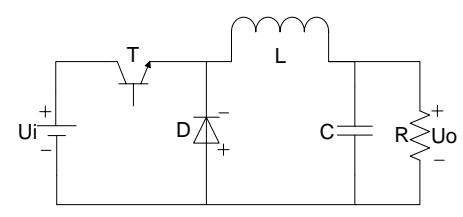

Fig.2 Topology of BUCK convertor

2.2 The analysis of working principle and the formula calculation

Under the condition of the input voltage $\mathrm{U}_{\mathrm{i}}$, output voltage $\mathrm{U}_{0}$, output power $\mathrm{P}_{0}$, rated frequency $\mathrm{f}_{s}$ are certain, the design method of BUCK converter working in CCM mode is as follows.

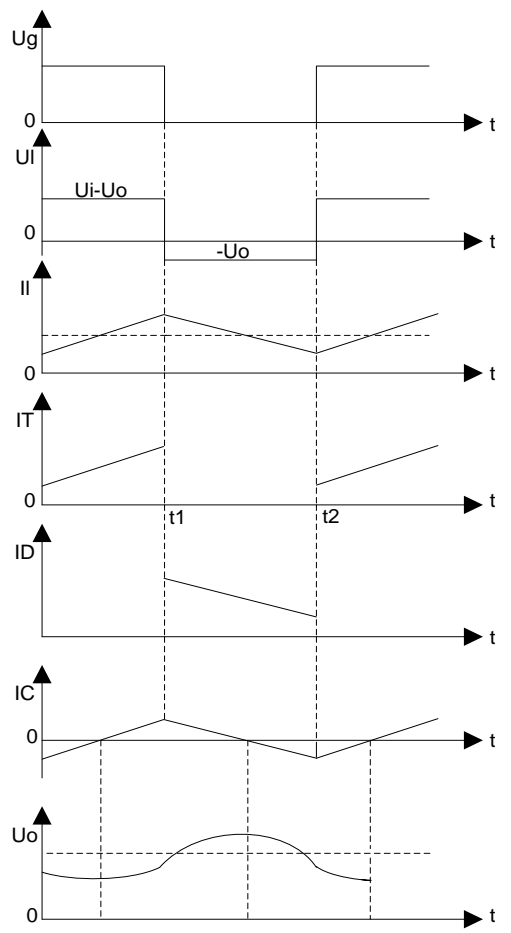

Fig.3Key waveforms of CCM mode 
(1)As shown in figure 3. When the switch tube $T$ is on, the fly-wheel diode $\mathrm{D}$ is cut off because of reverse biased. Capacitance $C$ starts charging and the input voltage Ui give energy to load through the inductor L. The inductance current I1 increases linearly and the stored magnetic energy also increases gradually. The current I0 flows through equivalent load $\mathrm{R}$. The output voltage of both ends is up positive and down negative. The conduction time of switch tube T is Ton in one Switching cycle TS.

(2)When the switch tube $\mathrm{T}$ is off, as the inductor current cannot change suddenly, I1 follows current through diode D. Inductor current decreases gradually and the energy on the inductance consume on the load step by step. I1 and the energy storage are reduced. When the inductor current is reducing, both ends of the inductive voltage $\mathrm{U} 1$ change polarity. The diode $\mathrm{D}$ is on under forward bias to constitute renewable flow loop. The output voltage of both ends are up positive and down negative. When $\mathrm{I}_{1}<\mathrm{I}_{0}$, capacitance is in discharge mode to keep Io and Uo unchanged. The switch tube broken time is Ts-Ton in one cycle Ts.

If the inductor current drops to zero at the moment Ts, it just comes to the critical state of continuous and discontinuous state. The relation between the load current I0 and I1 is :

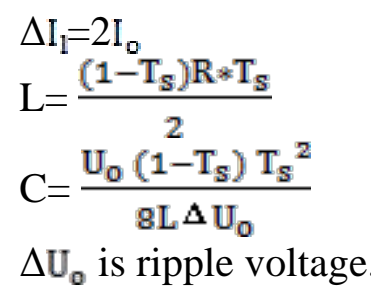

Assuming input voltage $\mathrm{Ui}=310 \mathrm{VM}$, output voltage $\mathrm{Uo}=180 \mathrm{~V}$, Rated switching frequency fs $=20 \mathrm{kHz}$ under rated load. After substituting these parameters into calculation formula above and correcting these parameters, the final parameter is: $\mathrm{L}=390 \mathrm{uH}, \mathrm{C}=110 \mathrm{uF}$.

After BUCK converter output dc voltage, the dc voltage will transmitted to full bridge inverter circuit. The function of full bridge converter is to invert dc power supply into ac square wave. The switching frequency of full bridge circuit is $15 \mathrm{kHz}$. Duty ratio is at maximum and remains the same. Dead band time is set as 16.7us. In this condition, the maximum voltage that the BUCK converter produced can fully transport to the final stage. The inverter circuit final stage accesses isolation transformer and the transformer variable ratio designs as 18:1. So that the transformer output voltage rating decreased to about $10 \mathrm{~V}$. Output current effective value can be up to $20 \mathrm{~A}$ according to the principle of power conservation. Output terminal accesses filament load to complete the role of the filament current eventually_producing electrons that electron beam melting furnace needs.

2.3The application of TMS320F28035

The choice of digital controller has a great impact on the power factor of switching power supply and the efficiency of system. This design uses TMS320F28035 type DSP of TI. As compared to the 28335 that has been widely used in power electronics industry, the main frequency of 28035 is only $60 \mathrm{MHz}$. But 28035 is added a CLA module. So control algorithm can be operated in the module separately. The programming can be easier. This design only needs 5 roads of PWM pulse, respectively transmitted to BUCK changes and parts of the full bridge inverter switch tube. Two roads of AD sampling will be needed to sampling the voltage and the current of BUCK convertor. DSP28035 can satisfies the function above completely and have a great advantage compared to DSP28335 on cost. So this design will take DSP28035 as the control chip.

\section{The Control Strategy}

The core of filament current control strategy is to control filament current at the steady state and heat the filament to produce electron. BUCK circuit has the advantage of small volume and simple control. This design adjusts the output of the filament current by adjusting the BUCK circuit duty ratios. Dc voltage after chopped by full bridge inverter unit is invert into ac voltage and transmitted to the buck isolation transformer.

As filament power supply, acceleration power supply and bombardment power supply are in series in EBM power system. Acceleration power supply can reach $30 \mathrm{kV}$ so the output voltage of filament 
power supply is extremely high. Sampling is very difficult and that is the reason that it is unable to realize closed loop control in the industrial production. In this design, current hall sensor is accessed to the output of BUCK converter to transform current signal into voltage signal. Then the signal will be accessed to the differential circuit to be disposed into $0 \sim 33 \mathrm{~V}$ voltage that can be transmitted to the AD sampling port of DSP28035 as the feedback signal. At the same time, convert the filament output current to transformer primary side as given signal that compared to the feedback signal. Error value will be sent to PI regulator for the current closed loop control.

\section{Simulation Analysis}

As shown in figrure3, Simulink model is established by the application of visualization simulation tool MATLAB. According to the calculated capacitance and inductance parameters above and putting the PI parameters into PI regulator, the power supply output voltage and current waveform can be got by the simulation.

As shown in figure 4, U0 is output voltage and I0 is output current. The output ac current can be $20 \mathrm{~A}$. Voltage effective value can reach $10 \mathrm{~V}$ under the condition of ideal resistance $0.5 \Omega$. The desired effect can be achieved under the condition of selecting the reasonable inductance, capacitance value and PI parameters.

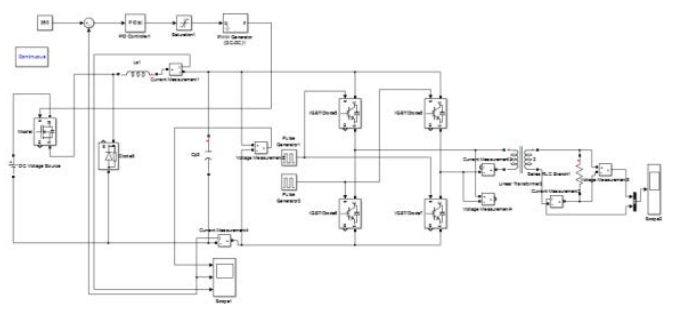

Fig.3 Simulation model of filament power supply

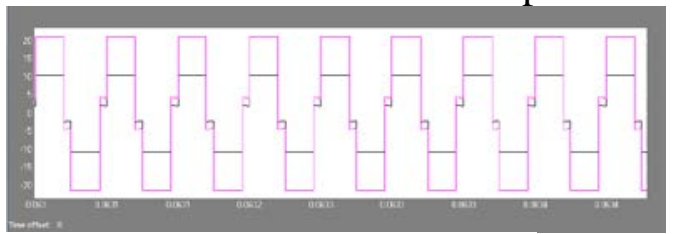

Fig.4 The output voltage and current waveform of filament power supply

\section{The Experimental Results}

Based on the theory above, this experiment designs a filament power supply prototype. The filament rated load is $0.5 \Omega$. Filament load is made up of 20 resistances of $10 \Omega$ in parallel. In the process of experiment, load resistance will change as the resistance wire temperature changes. The output current effective value remains at 20.2A. The output voltage effective value undulates from 8.3 to $16.5 \mathrm{~V}$. The main waveform under of load test is shown in Figure 5. CH1 and CH2 are the pulse waveform if Q1and Q3. Uo is output voltage and Io is output current.

Compared to the results of simulation, the output waveform is not ideal square wave because the resistance wire load is not pure resistance load and has some components of perceptual load. That is the reason the waveform component is indented. The process of the current waveform practical production has no obvious effects on the electron in the electron beam melting so the filament power can work normally. At the meantime, the temperature and the residence of the resistive load will rise along with the filament current increases. So the output voltage will increase gradually. 


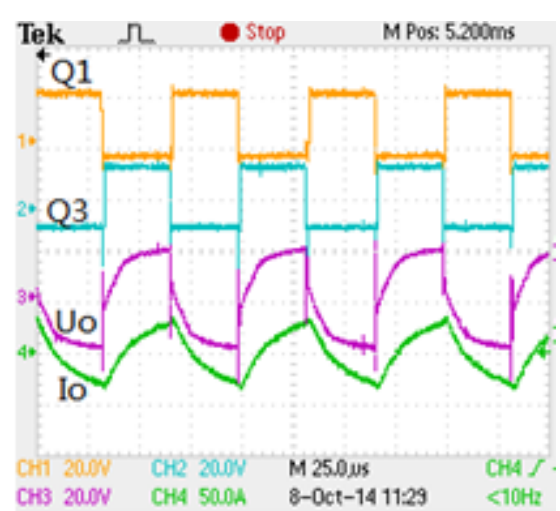

\section{Conclusion}

Fig.5 Key waveforms of experiment

The feasibility of using BUCK circuit as the filament power supply is verified by simulation on the purpose of realizing high frequency filament power supply and Closed-loop automatic adjustment in this article. The filament power supply system is simulated by using visualization simulation tool Simulink of MATLAB software. The output voltage and current waveform curve are given. Load test is done according to the simulation results. The experiment results show that the designed circuit parameters is reasonable and correct. It has important guiding significance for the electron beam melting furnace filament power supply high frequency and the development of automation.

\section{References}

[1] YIN Zhongrong, MA Yuan, ZHENG Jie. The research of the new type of electron beam melting furnace [J]. VACUUM, 2003, 11: 44-47.

[2]LIN Fei,DU Xin.The Matlib simulation of Power Electronies[M].Beijing: Electric power press of China, 2009.

[3] WEI Shouqi. The development of the heating power of the thermal type electron gun[J]. Beijing: New technology and new process 1997, 127: 8- 9.

[4] Zheng S, Czarkowski D. High-voltage high-power resonant converter for electrostatic precipitator[C]18th Annual IEEE Applied Power Electronics Conference and Exposition APEC. 2003, 3: 1100-1104.

[5] Kazimierczuk M K, Thirunarayan N, Wang S. Analysis of series-parallel resonant converter[J]. Aerospace and Electronic Systems, IEEE Transactions on, 1993, 29(1): 88-99.

[6] Liu J, Sheng L, Shi J, et al. LCC resonant converter operating under discontinuous resonant current mode in high voltage, high power and high frequency applications[C]//Applied Power Electronics Conference and Exposition, 2009. APEC 2009. Twenty-Fourth Annual IEEE. IEEE, 2009: 1482-1486. 